\title{
Serum Chemerin Predicts the Prognosis of Patients With Dilated Cardiomyopathy
}

\author{
Dan Chen, Juan Wang, Jianglin Fu \\ Department of Cardiovascular Medicine, Shiyan Renmin Hospital, Hubei University of Medicine, Hubei, China
}

\section{ABSTRACT}

Background: Chemerin is a newly discovered adipokine, which has been reported to be associated with the presence of dilated cardiomyopathy (DCM). The present study aims to evaluate the prognostic value of serum chemerin in patients with DCM.

Methods: A total of 214 patients with DCM was recruited and divided into 4 groups, according to quartiles of chemerin levels. Kaplan-Meier analysis was conducted to compare the survival rates among patients with different levels of chemerin, using the log-rank test. Multivariate Cox regression analysis was performed to assess the association of serum chemerin levels and occurrence of major adverse cardiac events (MACEs), including cardiac mortality, stroke and myocardial infarction.

Results: The Kaplan-Meier survival analysis indicated that patients with higher concentration of chemerin had shorter event-free survivals for MACEs $(P<.01)$. Cox regression analysis showed that chemerin was a significant predictor of MACEs (Quartile 3 versus Quartile 1: $\mathrm{HR}=1.79,95 \%$ CI: 1.31-2.79; Quartile 4 versus Quartile 1: $\mathrm{HR}=2.87,95 \%$ CI: 1.79-4.25) and all-cause death (Quartile 3 versus Quartile 1: $\mathrm{HR}=1.56$, 95\% CI: 1.20-2.42; Quartile 4 versus Quartile 1: $\mathrm{HR}=2.28,95 \% \mathrm{CI}: 1.52-3.96)$ after adjusting for potential risk factors.

Conclusion: Serum chemerin should be a potential prognostic indicator in patients with DCM.

\section{INTRODUCTION}

Dilated cardiomyopathy (DCM), one of the most common forms of cardiomyopathy and the main cause of heart failure, is characterized by ventricular chamber enlargement and systolic dysfunction with normal left ventricular wall thickness [Wu 2008; Maron 2006]. Despite a great deal of progress in the treatment of heart failure, the prognosis of most DCM patients remains poor $[\mathrm{Yu}$ 2016]. Therefore, effective biomarkers for individualized prediction of therapy outcomes and prognosis urgently are warranted.

Received fanuary 29, 2019; received in revised form March 21, 2020; accepted March 23, 2020.

Correspondence: Fianglin Fu, Department of Cardiovascular Medicine, Shiyan Renmin Hospital, No. 39 Chaoyang Central Road, Shiyan City, Hubei 442000, China;+86-0719-8637881 (e-mail: fujianglinsy@sobu.com).
Chemerin, a newly discovered adipokine, is expressed in different tissues such as the liver, pancreas, lung, and adipose tissues. It can regulate adipocyte differentiation and stimulate chemotaxis of dendritic cells and macrophages [Fatima 2014]. Increasing evidence has demonstrated that chemerin closely was associated with inflammation, obesity, metabolic syndrome, and coronary artery disease [Bozaoglu 2007; Gu 2014; Yan 2012; Xiaotao 2012]. Zhang et al reported that chemerin concentrations dramatically were increased in DCM compared with healthy controls and increased chemerin concentrations were associated with inflammation and left ventricular function in DCM [Zhang 2015]. However, it remains unclear whether chemerin is associated with the prognosis of DCM.

The present study aims to explore the prognostic value of serum chemerin in patients with DCM.

\section{METHODS}

Patients: Between January 2015 and June 2016, a total of 214 consecutive patients diagnosed with DCM at our hospital were recruited for this study. Eligible DCM patients were diagnosed based on the 1995 World Health Organization/ International Society and Federation of Cardiology (WHO/ ISFC) criteria [Richardson 1996]. Patients were excluded if they had previously known ischemic heart disease, congenital or valvular heart disease, myocarditis, and pericarditis. Patients with coexisting malignancy, acute infection, severe hepatic disease, sepsis, end-stage renal disease, and pregnancy also were excluded. All procedures involving human participants were performed in accordance with the ethical standards of the Institutional Review Board of our hospital and with the 1964 Declaration of Helsinki and its later amendments or comparable ethical standards. Informed consent was obtained from all individual participants included in the study.

Data collection: Demographic data, height, body weight, medical history, and medication use were obtained upon admission to hospital. Treatment strategies, including medication use and device therapy (implantable cardioverter defibrillator and cardiac resynchronization therapy implantation), were recorded during the follow up.

Blood samples were collected from all recruited DCM patients in a fasting state. Serum was obtained by centrifugation at $1000 \mathrm{~g}$ for 10 minutes and then stored at $-80^{\circ} \mathrm{C}$ until analysis. The concentrations of serum chemerin were measured using ELISA (R\&D Systems, USA), following the manufacturer's instructions. All samples were measured in duplicate. Chemerin levels were divided into quartiles. 
Table 1. Baseline characteristics of patients with DCM

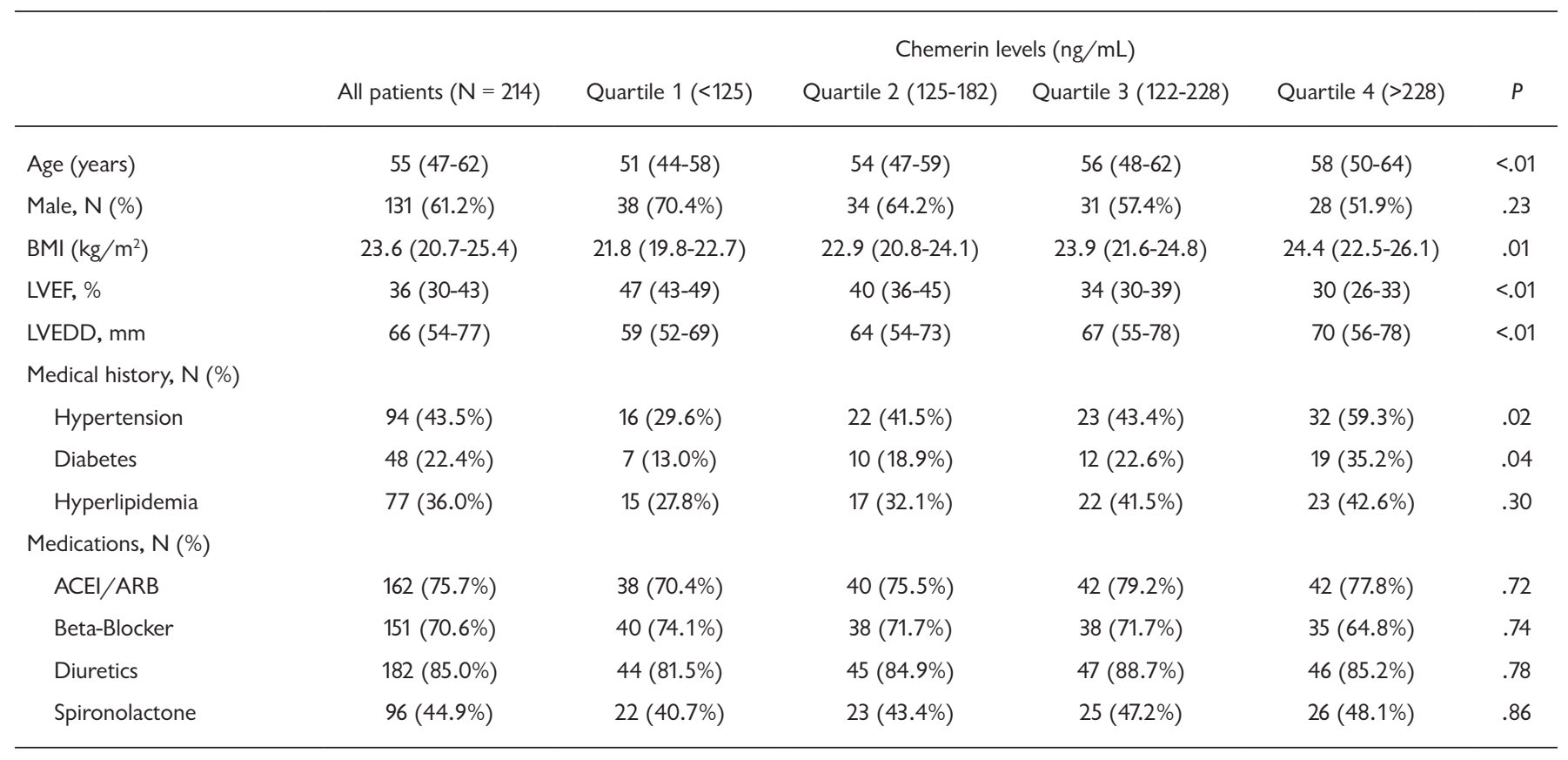

DCM, dilated cardiomyopathy; BMI, body mass index; LVEF, left ventricular ejection fraction; LVEDD, left ventricular end-diastolic diameter; ACEI, angiotensinconverting enzyme inhibitor; ARB, angiotensin receptor blocker.

Table 2. Association of chemerin levels with the risk of MACEs in patients with DCM

\begin{tabular}{lcccc}
\hline & & \multicolumn{2}{c}{ Chemerin levels (ng/mL) } \\
& Quartile 1 $(<125)$ & Quartile 2 (125-182) & Quartile 3 (122-228) & Quartile 4 (>228) \\
\hline Unadjusted model & 1 & $1.28(0.79-1.86)$ & $2.24(1.47-3.18)$ & $3.47(2.09-5.07)$ \\
Adjusted model 1 & 1 & $1.24(0.73-1.78)$ & $1.99(1.38-2.82)$ & $3.16(1.97-4.48)$ \\
Adjusted model 2 & 1 & $1.17(0.68-1.67)$ & $1.79(1.31-2.79)$ & $2.87(1.79-4.25)$ \\
Adjusted model 3 & 1 & $1.19(0.70-1.72)$ & $1.81(1.35-2.82)$ & $2.99(1.85-4.28)$ \\
\hline
\end{tabular}

Adjusted model 1: adjusted for age, sex and BMl;

Adjusted model 2: adjusted for demographic variables (age, sex and BMI), medical history, medications, LVEF and LVEDD.

Adjusted model 3: adjusted for demographic variables (age, sex and BMI), medical history, medications, LVEF, LVEDD and treatments during follow up

Primary and secondary endpoints: The primary endpoint was occurrence of major adverse cardiac events (MACEs), including cardiac mortality, stroke, and myocardial infarction. The secondary endpoint was the occurrence of all-cause mortality. Endpoints were obtained by reviewing the hospital records and contacting patients or their families.

Statistical analysis: Continuous variables were presented as median and interquartile range and compared by nonparameter test. Categorical variables were presented as proportions and compared with the Chi-square test or Fisher's exact test. Kaplan-Meier analysis was undertaken to compare the survival rate among patients with different levels of chemerin using the log-rank test. Multivariate Cox proportional hazards regression analysis was performed to assess the association of serum chemerin and risk of MACEs, adjusting for potential confounders. All tests were 2 -sided and a $\mathrm{P}$ value of less than 0.05 was considered significant.

All statistical analyses were performed with the SPSS statistical software program package (SPSS version 20.0 for Windows, SPSS Inc., Chicago, Illinois, USA).

\section{RESULTS}

Patients' characteristics are presented in Table 1. All patients with DCM were categorized into 4 groups, according to quartiles of serum chemerin concentration. It is shown that patients with higher chemerin levels were older $(P<.01)$ 
Table 3. Association of chemerin levels with the risk of all-cause death in patients with DCM

\begin{tabular}{lcccc}
\hline & & \multicolumn{2}{c}{ Chemerin levels (ng/mL) } \\
& Quartile 1 $(<125)$ & Quartile 2 (125-182) & Quartile 3 (122-228) & Quartile 4 (>228) \\
\hline Unadjusted model & 1 & $1.32(0.63-1.81)$ & $1.91(1.40-2.98)$ & $3.02(2.19-4.76)$ \\
Adjusted model 1 & 1 & $1.22(0.74-1.50)$ & $1.77(1.38-2.24)$ & $2.57(1.74-4.21)$ \\
Adjusted model 2 & 1 & $1.18(0.72-1.50)$ & $1.66(1.26-2.58)$ & $2.39(1.58-4.03)$ \\
Adjusted model 3 & 1 & $1.20(0.75-1.54)$ & $1.71(1.33-2.64)$ & $2.44(1.60-4.15)$ \\
\hline
\end{tabular}

Adjusted model 1: adjusted for age, sex and BMI;

Adjusted model 2: adjusted for demographic variables (age, sex and BMI), medical history, medications, LVEF and LVEDD.

Adjusted model 3: adjusted for demographic variables (age, sex and BMI), medical history, medications, LVEF, LVEDD and treatments during follow up.

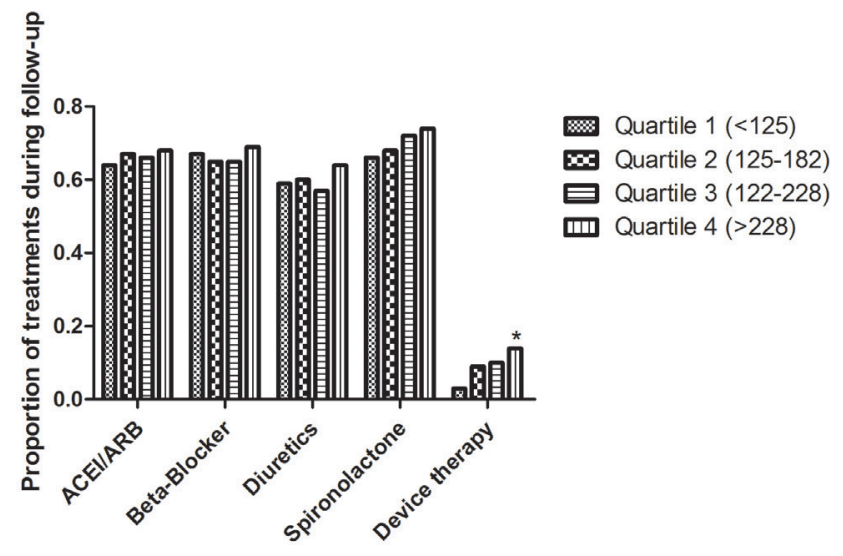

Figure 1. The proportion of pharmaceutical and device therapy during follow up. ${ }^{*} P<.01$ compared with Quartile 1 group. (ACEl, angiotensin-converting enzyme inhibitor; ARB, angiotensin receptor blocker).

and were more likely to experience hypertension $(P=.02)$ and diabetes $(P=.04)$. Chemerin levels positively were associated with body mass index (BMI; $P=.01)$ and left ventricular enddiastolic diameter (LVEDD; $P<.01)$ and were inversely associated with left ventricular ejection fraction (LVEF; $P<.01$ ).

The median length of follow up was 18 months. The proportion of pharmaceutical and device therapies during the follow up were displayed in Figure 1. (Figure 1) The proportion of pharmaceutical therapies were similar among the 4 groups, while the proportion of device therapy was significantly higher $(P<.01)$ for patients in chemerin Quartile 4 group compared with those in Quartile 1 group.

Among the 214 enrolled DCM patients, 36 (16.8\%) patients died, $66(30.8 \%)$ patients suffered from MACEs, and $112(52.3 \%)$ patients censored. No patients were lost to follow up. As shown in Figure 2, the Kaplan-Meier survival curve displayed that patients with higher concentration of chemerin had shorter event-free survival for MACEs $(P<.01)$ (Figure 2$)$.

Multivariate Cox proportional hazards regression analyses are performed to explore the association of serum chemerin levels with the risk of MACEs in patients with

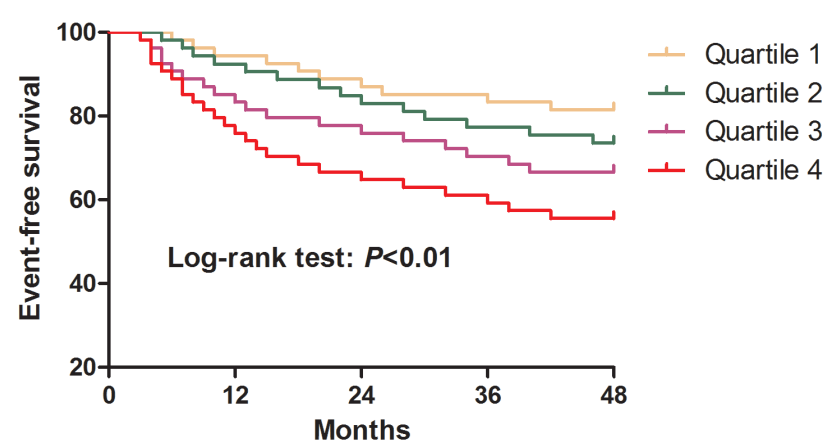

Figure 2. Kaplan-Meier survival analysis. The event-free survival for major adverse cardiac events (MACEs) in patients with dilated cardiomyopathy (DCM), stratified by the quartiles of chemerin concentrations.

DCM (Table 2). It is shown that after adjusting for demographic variables (age, sex, and BMI), medical history, medications, LVEF, LVEDD and treatments during follow up, serum chemerin concentrations were positively associated with the risk of MACEs (Quartile 3 versus Quartile 1: HR = 1.81, 95\% CI: 1.35-2.82; Quartile 4 versus Quartile 1: HR = 2.99, $95 \%$ CI: $1.85-4.28)$.

The association of serum chemerin levels with the risk of all-cause death was shown in Table 3 . It is indicated that the elevated serum chemerin levels were related to an increase risk of all-cause death (Quartile 3 versus Quartile 1: HR = 1.71, 95\% CI: 1.33-2.64; Quartile 4 versus Quartile 1: HR = 2.44, 95\% CI: 1.60-4.15) after adjusting for potential confounders.

\section{DISCUSSION}

Though DCM shows overall a favorable cardiac recovery, a substantial proportion of patients suffer from persistent heart failure leading to increased mortality and heart transplantation [McNamara 2011]. Thus, identification of highrisk DCM patients is important, and biomarkers widely are employed to either detect or manage diseases. The present study revealed that patients with higher concentration of 
chemerin had longer event-free survivals for MACEs and allcause death, indicating that chemerin was a significant predictor of prognosis in patients with DCM.

A previous study conducted by Zhang et al [Zhang 2015] showed that chemerin concentrations significantly were higher in DCM patients compared with the control group. The multivariate logistic regression analysis reported that chemerin independently was associated with the presence of DCM $(\mathrm{OR}=1.102,95 \%$ CI: $1.052-1.153 ; P<.01)$. Several other clinical studies also have demonstrated the relationship between chemerin and cardiovascular diseases (CAD). Menzel et al showed that participants in the fourth quartile of chemerin had a more than fourfold higher risk of heart failure [Menzel 2017]. Leiherer et al investigated the association between chemerin and cardiovascular endpoints in 495 patients undergoing coronary angiography for the evaluation of established or suspected stable CAD, showing that patients with high chemerin concentrations were more often affected by cardiovascular events, defined by vascular deaths, nonfatal myocardial infarctions, non-fatal strokes, and the necessity of cardiovascular intervention [Leiherer 2016]. However, few studies explored the prognostic value of chemerin in the patients with cardiovascular diseases. Zhou et al investigated the association between serum chemerin and clinical outcomes in 834 patients with heart failure, during a median follow up of 524 days. The multivariate Cox regression analysis showed that after adjustment for demographic variables, traditional risk factors, estimated glomerular filtration rate and highsensitivity Creactive protein, serum chemerin remained a significant predictor of MACEs in patients with heart failure [Zhou 2019]. Our study firstly reported that serum chemerin should be a prognostic predictor in patients with DCM.

So far, the mechanisms of chemerin and cardiovascular pathologies are still unclear. A recent study by RodríguezPenas et al proposed possible chemerin-related pathways on viability in murine cardiomyocytes [Rodriguez-Penas 2015], which may provide possible explanation for the association between higher chemerin concentrations and increased risk of MACEs in the present study. It observed that chemerin induced apoptosis directly in cultured cardiomyocytes in a dose- and time-dependent manner. Moreover, chemerin partially suppresses AKT (protein kinase B) phosphorylation at Thr308, which has been linked to apoptosis. It is known that apoptosis is a tightly regulated, cell deletion process that plays an important role in various cardiovascular diseases, such as myocardial infarction, reperfusion injury, and heart failure [Kim 2010]. Apart from that, Zhang et al reported that chemerin concentrations positively were associated with inflammation indicated by increased concentrations of IL-6, TNF- $\alpha$, and C-reactive protein (CRP)) and caused cardiac dysfunction, which also may be a reason for the poor prognosis in patients with higher serum chemerin [Zhang 2015]. Nevertheless, the exact mechanisms require further exploration.

Our study had several limitations. First, the present findings are based on single measurement of chemerin and serial measurements were not performed during the follow-up period. In addition, some clinical indicators, such as New
York Heart Association (NYHA) functional class, are not collected, which may cause bias.

In conclusion, our study demonstrated that higher serum chemerin was associated with an increased risk of MACEs, which may serve as a prognostic indicator in patients with DCM.

\section{REFERENCES}

Bozaoglu K, Bolton K, McMillan J, et al. 2007. Chemerin is a novel adipokine associated with obesity and metabolic syndrome. Endocrinology 148(10):4687-94.

Fatima SS, Rehman R, Baig M, et al. 2014. New roles of the multidimensional adipokine: chemerin. Peptides 62:15-20.

Gu P, Jiang W, Lu B, et al. 2014. Chemerin is associated with inflammatory markers and metabolic syndrome phenotypes in hypertension patients. Clin Exp Hypertens 36(5):326-32.

Kim NH, Kang PM. 2010. Apoptosis in cardiovascular diseases: mechanism and clinical implications. Korean Circ J 40(7):299-305.

Leiherer A, Muendlein A, Kinz E, et al. 2016. High plasma chemerin is associated with renal dysfunction and predictive for cardiovascular events - Insights from phenotype and genotype characterization. Vascul Pharmacol 77:60-8.

Maron BJ, Towbin JA, Thiene G, et al. 2006. Contemporary definitions and classification of the cardiomyopathies: an American Heart Association Scientific Statement from the Council on Clinical Cardiology, Heart Failure and Transplantation Committee; Quality of Care and Outcomes Research and Functional Genomics and Translational Biology Interdisciplinary Working Groups; and Council on Epidemiology and Prevention. Circulation 113(14):1807-16.

McNamara DM, Starling RC, Cooper LT, et al. 2011. Clinical and demographic predictors of outcomes in recent onset dilated cardiomyopathy: results of the IMAC (Intervention in Myocarditis and Acute Cardiomyopathy)-2 study. J Am Coll Cardiol 58(11):1112-8.

Menzel J, di Giuseppe R, Biemann R, et al. 2017. Association between chemerin, omentin-1 and risk of heart failure in the population-based EPIC-Potsdam study. Sci Rep 7(1):14171.

Richardson P, McKenna W, Bristow M, et al. 1996. Report of the 1995 World Health Organization/International Society and Federation of Cardiology Task Force on the Definition and Classification of cardiomyopathies. Circulation 93(5):841-2.

Rodriguez-Penas D, Feijoo-Bandin S, Garcia-Rua V, et al. 2015. The Adipokine Chemerin Induces Apoptosis in Cardiomyocytes. Cell Physiol Biochem 37(1):176-92.

Wu T, Chen Y, Du Y, et al. 2008. Serum Exosomal MiR-92b-5p as a Potential Biomarker for Acute Heart Failure Caused by Dilated Cardiomyopathy. Cell Physiol Biochem 46(5):1939-50.

Xiaotao L, Xiaoxia Z, Yue X, et al. 2012. Serum chemerin levels are associated with the presence and extent of coronary artery disease. Coron Artery Dis 23(6):412-6.

Yan Q, Zhang Y, Hong J, et al. 2012. The association of serum chemerin level with risk of coronary artery disease in Chinese adults. Endocrine 41(2):281-8.

Yu M, Liang W, Xie Y, et al. 2016. Circulating miR-185 might be a novel biomarker for clinical outcome in patients with dilated cardiomyopathy. 
Sci Rep 6:33580.

Zhang O, Ji Q, Lin Y, et al. 2015. Circulating chemerin levels elevated in dilated cardiomyopathy patients with overt heart failure. Clin Chim
Acta 448:27-32.

Zhou X, Tao Y, Chen Y, et al. 2019. Serum Chemerin as a Novel Prognostic Indicator in Chronic Heart Failure. J Am Heart Assoc 8(15):e012091. 